\title{
ESTIMATIONS OF POWER DIFFERENCE MEAN BY HERON MEAN
}

\section{MASATOSHI ITO}

Abstract. As generalizations of arithmetic and geometric means, for positive real numbers $a$ and $b$, power difference means $J_{q}(a, b)=\frac{q}{q+1} \frac{a^{q+1}-b^{q+1}}{a^{q}-b^{q}}$ and Heron means $K_{q}(a, b)=(1-q) \sqrt{a b}+$ $q \frac{a+b}{2}$ are well known. In this paper, we obtain the greatest value $\alpha=\alpha(q)$ and the least value $\beta=\beta(q)$ such that the double inequality

$$
K_{\alpha}(a, b)<J_{q}(a, b)<K_{\beta}(a, b)
$$

holds for any $q \in \mathbb{R}$, which includes Xia, Hou, Wang and Chu's result. Moreover, from this result, we derive operator inequalities for bounded linear operators on a Hilbert space.

Mathematics subject classification (2010): Primary 26E60, 47A63.

Keywords and phrases: Power difference mean, Heron mean and operator mean.

\section{REFERENCES}

[1] R. BHATIA, Interpolating the arithmetic-geometric mean inequality and its operator version, Linear Algebra Appl., 413 (2006), 355-363.

[2] J. I. FujII AND Y. Seo, On parameterized operator means dominated by power ones, Sci. Math., 1 (1998), 301-306.

[3] M. FujiI, S. FuRUichi AND R. NAKAmoto, Estimations of Heron means for positive operators, J. Math. Inequal., 10 (2016), 19-30.

[4] S. FURUICHI, Operator inequalities among arithmetic mean, geometric mean and harmonic mean, J. Math. Inequal., 8 (2014), 669-672.

[5] S. Furuichi And K. Yanagi, Bounds of the logarithmic mean, J. Inequal. Appl. 2013, 2013:535, $11 \mathrm{pp}$.

[6] T. FURUTA, Concrete examples of operator monotone functions obtained by an elementary method without appearing to Löwner integral representation, Linear Algebra Appl., 429 (2008), 972-980.

[7] Z.-Y. HE, M.-K. WANG AND Y.-M. ChU, Optimal one-parameter mean bounds for the convex combination of arithmetic and logarithmic means, J. Math. Inequal., 9 (2015), 699-707.

[8] F. Hiai And H. Kos AKI, Comparison of various means for operators, J. Funct. Anal., 163 (1999), 300-323.

[9] F. HIAI AND H. Kos AKI, Means for matrices and comparison of their norms, Indiana Univ. Math. J., 48 (1999), 899-936.

[10] F. Kubo And T. Ando, Means of positive linear operators, Math. Ann., 246 (1980), 205-224.

[11] Y. UDAGAWA, S. WADA, T. YAMAZAKI AND M. YANAGIDA, On a family of operator means involving the power difference means, Linear Algebra Appl., 485 (2015), 124-131.

[12] W.-F. XiA, S.-W. Hou, G.-D. WANG AND Y.-M. ChU, Optimal one-parameter mean bounds for the convex combination of arithmetic and geometric means, J. Appl. Anal., 18 (2012), 197-207.

[13] W.-F. XIA, G.-D. WANG, Y.-M. ChU AND S.-W. Hou, Sharp inequalities between one-parameter and power means, Adv. Math. (China), 42 (2013), 713-722. 